\title{
O CONHECIMENTO DO DIAGNÓSTICO DE CÂNCER NÃO LEVA À DEPRESSÃO EM PACIENTES SOB CUIDADOS PALLATIVOS
}

Renata Wanderley Diniz, Marina Sahade Gonçalves, Carolina Games Bensi*, Arinilda Silva Campos, Auro del Giglio, Juliana Bueno Garcia, Vanessa da Costa Miranda, Tatiana Alves Monteiro, Michelle Rosemberg

Trabalho realizado na Faculdade de Medicina do ABC e Instituto de Oncologia - Sociedade Acadêmica de Estudo e Controle do Câncer, Santo André, SP

* Correspondência:

Rua Luís Fiorotti, 657

09570-030, São Caetano

do Sul, SP

\begin{abstract}
RESUMO
Oвjetivos. Traçar o perfil e identificar a prevalência de depressão nos pacientes sob cuidados paliativos no Serviço de Oncologia da Faculdade de Medicina do ABC.

Métodos. Entrevistamos 62 pacientes oncológicos em cuidados paliativos que responderam a três questionários: questionário geral contendo variáveis demográficas, questionário estruturado para pacientes oncológicos em cuidados terminais para avaliação de sua qualidade de vida e inventário de depressão de Beck.

Resultados. Observamos que 68\% dos pacientes tinham algum grau de depressão. A maioria dos pacientes sabia seu diagnóstico (87,1\%), estava satisfeito com o tratamento (93,33\%), sentia-se satisfeito com o apoio recebido (95,7\%) e referiu não conversar com seus médicos sobre outros assuntos além de sua saúde (81,18\%). Os sintomas mais freqüentes foram dor, cansaço, fraqueza e alterações do sono. Encontramos que o fato de não saber o diagnóstico $(p=0,008)$, estar internado $(p=0,0019)$ e não ter recebido tratamento oncológico $(p=0,007)$ se correlacionam significativamente com níveis mais altos de depressão.
\end{abstract}

Conclusão. Pacientes sob cuidados paliativos em nosso meio, apesar de geralmente satisfeitos com seu cuidado, relatam pobre comunicação com seu médico e apresentam uma alta taxa de depressão. Saber o seu diagnóstico e ter recebido tratamento oncológico se correlacionam inversamente com a presença de depressão.

UnIteRmos: Câncer. Assistência paliativa. Depressão. Qualidade de vida.

\section{INTRODUÇÃO}

O câncer é uma doença que está entre as principais causas de morte, constituindo atualmente no Brasil a segunda causa de mortalidade, sendo superado apenas pelas doenças cardiovasculares'. Segundo o Instituto Nacional de Câncer, a incidência no Brasil é de aproximadamente 400.000 novos casos por ano, sendo que destes cerca de 127.000 evoluem a óbito'. Nos EUA, esses números são ainda maiores, sendo que para o ano de 2004 a estimativa foi de 1.368 .000 novos casos e 563.700 óbitos $^{2}$.

Apesar dos grandes avanços no tratamento oncológico, nem sempre é possível obter a cura; com isso, muitos pacientes passam a necessitar de cuidados que visam, além do controle da dor e de outros sintomas diversos, interferir nos aspectos psicológicos, sociais e espirituais, no intuito de investir na melhora de sua qualidade de vida ${ }^{3}$.

O termo "palliare" tem origem no latim e significa proteger, amparar, cobrir, abrigar, ou seja, a perspectiva do cuidar e não somente curar. De acordo com a Organização Mundial da Saúde (OMS), cuidados paliativos são definidos como cuidados ativos e totais aos pacientes quando a doença não responde aos tratamentos curativos, e o controle de sintomas torna-se prioridade, objetivando melhor qualidade de vida para pacientes e familiares ${ }^{5}$. Já o conceito de qualidade de vida pode ser definido como uma avaliação global que o sujeito faz da sua vida, e depende tanto das características do sujeito (demográficas, personalidade, valores, etc) como de moduladores externos, como a doença, seus sintomas e o tratamento que esta requer 5 .

A depressão é comum em pacientes com câncer em estágio terminal e pode ser uma importante fonte de angústia tanto para o paciente como para seus parentes ${ }^{6,7}$. A importância de se diagnosticar a depressão em pacientes sob cuidados paliativos deve-se ao fato de que, além do comprometimento emocional, os pacientes deprimidos também apresentam sintomas físicos que são difíceis de "paliar" e que podem ser facilmente aliviados quando a depressão é tratada adequadamente, com a conseqüente melhora da qualidade de vida deste indivíduo ${ }^{7}$.

Apesar dos benefícios observados com o tratamento da depressão, esta ainda permanece freqüentemente subdiagnosticada $8,9,10$. De fato, Maguire (1985) mostrou que mais de 80\% das comorbidades psicológicas e psiquiátricas presentes nos pacientes oncológicos passam despercebidas e não são tratadas".

Acredita-se que uma explicação para esta baixa taxa de detecção dever-se-ia a não comunicação efetiva do paciente com seu médico, por achar que, de alguma forma, seria o culpado pela sua angústia ou que estaria desperdiçando o tempo do profissional e, portanto, optaria por esconder seus verdadeiros sentimentos ${ }^{12}$.

$\mathrm{Na}$ literatura brasileira, ainda são poucos os estudos que enfocam o paciente oncológico em cuidados paliativos, suas necessidades, sua qualidade de vida e a presença de depressão nesta fase de sua vida. 
O objetivo deste estudo é traçar o perfil e identificar a prevalência de depressão nos pacientes sob cuidados paliativos do Serviço de Oncologia da Faculdade de Medicina do ABC (FMABC) e, a partir dos dados encontrados, sugerir condições que melhorem a abordagem e conseqüentemente a qualidade de vida destes pacientes.

\section{Métodos}

Foi realizado um estudo transversal observacional e prospectivo, envolvendo pacientes oncológicos em tratamento paliativo nos ambulatórios ou internados nos hospitais ligados à Faculdade de Medicina do ABC no período de janeiro a maio de 2005.

O estudo foi realizado no Hospital Estadual Mário Covas, Hospital de Ensino Padre Anchieta e Instituto de Oncologia da Faculdade de Medicina do $A B C$, tendo sido aprovado pela Comissão de Ética em Pesquisa da FMABC em novembro de 2004. Foram entrevistados 62 pacientes, sendo que $4 \mathrm{I}$ estavam em atendimento ambulatorial e 21 internados, num período de cinco meses. Os entrevistados foram escolhidos com base nos seguintes critérios de inclusão:

- Presença de neoplasia maligna diagnosticada;

- Em regime de internação hospitalar nos hospitais ligados a FMABC ou em acompanhamento nos ambulatórios de oncologia desta instituição;

- Sob cuidados paliativos devido à doença neoplásica;

- Ausência de tratamento antineoplásico com intuito curativo por ocasião da aplicação do questionário;

- Idade superior ou igual a 18 anos;

- Deviam estar orientados e contactuantes;

- Concordassem em participar no estudo por meio da assinatura do termo de consentimento livre e esclarecido.

Neste trabalho, o conceito de cuidados paliativos baseia-se na definição da OMS, que os considera como cuidados ativos e totais quando a doença não responde aos tratamentos curativos, priorizando o controle dos sintomas com o objetivo de melhorar a qualidade de vida do paciente ${ }^{5}$.

Após ser explicado o objetivo do estudo, era solicitado o consentimento do paciente e, então, a entrevista era realizada por um estudante de medicina previamente treinado. A entrevista consistia em três questionários, explicados a seguir.

\section{Questionários}

I. Questionário geral - composto por variáveis descritivas demográficas, com informações sobre sua internação e doença.

2. Questionário estruturado para pacientes oncológicos em cuidados paliativos (baseado no PQLI - Paliattive Care Quality of Life Instrument) - composto por 28 itens sobre a qualidade de vida na última semana, divididos em oito subgrupos: atividades, autonomia, saúde, escolha do tratamento, apoio, comunicação, efeitos psicológicos e qualidade de vida global. Este questionário não foi validado para a língua portuguesa, portanto não tentamos derivar dele nenhum tipo de pontuação e o utilizamos apenas como um elenco de quesitos pertinentes a pacientes em cuidados paliativos somente para traçar qualitativamente um perfil do paciente $^{13}$.
3. Inventário de depressão de BECK - composto por 21 itens, cuja escala varia de 0 a 3. Estes referem-se a tristeza, pessimismo, sensação de fracasso, culpa e punição; falta de satisfação; autodepreciaçãa; auto-acusações; idéias suicidas; crises de choro; irritabilidade; retração social; indecisão; distorção da imagem corporal; inibição para o trabalho; distúrbio do sono; fadiga; perda de apetite e de peso; preocupação somática e diminuição de libido. A análise do Inventário de depressão de Beck considera os escores de 0 a 9 como ausência de depressão, de 10 a 18 depressão leve, de 19 a 29 depressão moderada, e de 30 a 63 depressão grave. Utilizamos a versão deste questionário já validado para uso em português ${ }^{14}$.

\section{Métodos estatísticos}

Para a estatística descritiva utilizamos o programa Microsoft Excel (Windows Millenium Edition). Para a análise estatística utilizamos o programa NCSS 2000-PASS 2000 (Utah, EUA). Na análise das variáveis discretas utilizamos o teste Qui-quadrado ou Fisher, quando necessário. Para comparação de médias utilizamos o método Anova. Consideramos significativos valores de $p$ iguais ou menores que 0,05 .

\section{Resultados}

Participaram do estudo 62 pacientes oncológicos em tratamento paliativo nos ambulatórios ou internados nos hospitais ligados à Faculdade de Medicina do ABC, sendo 26 (41,9\%) do sexo masculino e 36 (58, $1 \%$ ) do sexo feminino. A média de idade encontrada foi de 58,6 anos, variando de 26 a 88 (Tabela I).

Entre os pacientes entrevistados, $4 \mathrm{I}(66,13 \%)$ estavam sob cuidados ambulatoriais e 21 (33,87\%) encontravam-se internados. Com relação aos dados clínicos, foi encontrado que 58,06\% dos pacientes apresentavam alguma comorbidade, além do câncer (Tabela I). O tumor primário mais freqüente foi o de mama (32,26\%), seguido por pulmão, laringe e estômago com uma porcentagem de 8,06\%; os demais sítios correspondem a 40,32\%. O tempo do diagnóstico foi em média de 41,32 meses, variando de 0,25 a 180 meses. Cinqüenta e três pacientes $(85,48 \%)$ apresentavam tumor sabidamente metastático (Tabela I). Os demais dados demográficos dos pacientes se encontram na Tabela I.

Dentre os pacientes, 54 (87, I\%) sabiam de seu diagnóstico, sendo que 31 (50\%) consideravam-se informados sobre a doença e sem dúvidas sobre a mesma (Tabela 2).

Cinqüenta e oito $(93,55 \%)$ pacientes foram submetidos a algum tipo de tratamento. Sobre a decisão de parar de recebê-lo, 30 $(61,22 \%)$ referiram não ter participado desta escolha. A grande maioria dos pacientes (93,33\%) estava satisfeita com o tratamento recebido (Tabela 2). De fato, quanto à escolha do tratamento, a maioria dos entrevistados não gostaria de escolher seu esquema terapêutico (68,85\%) e nem se consideraria capaz para tal (73,77\%). O aspecto 
Tabela I - Características demográficas: aspectos gerais dos pacientes sob cuidados paliativos

\begin{tabular}{|c|c|}
\hline Características & $\mathbf{N}(\%)$ \\
\hline \multicolumn{2}{|l|}{ Atendimento } \\
\hline Hospitalar(internado) & $21(33,87 \%)$ \\
\hline Ambulatorial & $41(66,13 \%)$ \\
\hline \multicolumn{2}{|l|}{ Sexo } \\
\hline Masculino & $26(41,90 \%)$ \\
\hline Feminino & $36(58,10 \%)$ \\
\hline \multicolumn{2}{|l|}{ Estado Civil } \\
\hline Solteiro/separado & $16(25,80 \%)$ \\
\hline Casado/amasiado & $33(53,23 \%)$ \\
\hline Viúvo & $13(20,97 \%)$ \\
\hline \multicolumn{2}{|l|}{ Escolaridade } \\
\hline Analfabeto & $10(16,40 \%)$ \\
\hline Atéfundamental completo & $40(65,60 \%)$ \\
\hline Até médio completo & $7(\mid 1,50 \%)$ \\
\hline Até superior completo & $4(6,50 \%)$ \\
\hline \multicolumn{2}{|l|}{ Religião } \\
\hline Católica & $40(65,57 \%)$ \\
\hline Protestante & | (1,63\%) \\
\hline Budista & $0(0 \%)$ \\
\hline Espirita & $2(3,27 \%)$ \\
\hline Evangélico & $17(27,90 \%)$ \\
\hline Outros & I (I,63\%) \\
\hline \multicolumn{2}{|l|}{ Atividade religiosa } \\
\hline Não Praticante & $25(42,37 \%)$ \\
\hline Praticante & $34(57,63 \%)$ \\
\hline \multicolumn{2}{|l|}{ Provedor da casa } \\
\hline Paciente & $23(37,10 \%)$ \\
\hline Outros & $39(62,90 \%)$ \\
\hline \multicolumn{2}{|l|}{ Tem família } \\
\hline Sim & $62(100 \%)$ \\
\hline Não & $0(0 \%)$ \\
\hline \multicolumn{2}{|l|}{ Mora com quem } \\
\hline Sozinho(a) & $6(9,68 \%)$ \\
\hline Com outras pessoas & $56(90,32 \%)$ \\
\hline \multicolumn{2}{|l|}{ Comorbidade } \\
\hline Não & $26(41,93 \%)$ \\
\hline $\operatorname{Sim}$ & $36(58,07 \%)$ \\
\hline \multicolumn{2}{|l|}{ Sítio do tumor primário } \\
\hline Mama & $20(32,26 \%)$ \\
\hline Laringe & $5(8,06 \%)$ \\
\hline Pulmão & $5(8,06 \%)$ \\
\hline Estômago & $5(8,06 \%)$ \\
\hline Outros & $25(40,32 \%)$ \\
\hline Não sabe sítio & $2(3,23 \%)$ \\
\hline \multicolumn{2}{|l|}{ Tempo do diagnóstico (meses) } \\
\hline Média & 41,32 \\
\hline Valor máximo - mínimo & $0,25-180$ \\
\hline \multicolumn{2}{|l|}{ Metástase } \\
\hline $\operatorname{Sim}$ & $53(85,48 \%)$ \\
\hline Não & $6(9,68 \%)$ \\
\hline Não sabe & $3(4,84 \%)$ \\
\hline
\end{tabular}

Tabela 2 - Características clínicas e do tratamento dos pacientes sob cuidados paliativos

\begin{tabular}{lc}
\hline & \multicolumn{1}{c}{ Características } \\
\cline { 2 - 2 } Diagnóstico & $\mathbf{N}(\%)$ \\
Sabe & $54(87,10 \%)$ \\
Não sabe & $8(12,90 \%)$ \\
Informações que tem sobre a doença & \\
Não sabe e não quer saber & $6(9,67 \%)$ \\
Não sabe e quer saber mais & $10(16,13 \%)$ \\
Sabe e tem dúvida & $15(24,20 \%)$ \\
Sabe e não tem dúvida & $31(50,00 \%)$ \\
Recebeu tratamento & \\
Sim & $58(93,55 \%)$ \\
Não & $4(6,45 \%)$ \\
Tipo de tratamento & \\
Quimioterapia & $43(75,44 \%)$ \\
Radioterapia & $6(10,53 \%)$ \\
Cirurgia & $5(8,77 \%)$ \\
Hormônio & $5(8,77 \%)$ \\
Participou da decisão de parar de tratar & \\
Sim & $19(38,78 \%)$ \\
Não & $30(61,22 \%)$ \\
Satisfeito com o tratamento & \\
Sim & $56(93,33 \%)$ \\
Não & $4(6,67 \%)$ \\
\hline
\end{tabular}

considerado mais importante na escolha do tratamento foi a qualidade de vida (45, I5\%), seguido pela eficácia do tratamento $(40,32 \%)$ (Tabela 3).

Com relação ao questionário estruturado para pacientes oncológicos em tratamento paliativo (PQLI), foi encontrado que a maior parte dos pacientes não conseguia realizar suas atividades diárias (60\%), julgava-se auto-suficiente para realizar tarefas como ir ao banheiro, vestir-se, entre outros $(68,55 \%)$. Os sintomas mais freqüentes na nossa amostra foram dor (67,74\%), cansaço/fraqueza (56,3\%) e alterações do sono (66,l\%). A maior parte dos pacientes sentia-se satisfeita com o apoio de familiares, amigos, equipe médica e enfermagem (95,7\%). A maioria dos pacientes referia não conversar com seus médicos sobre outros assuntos além de sua condição de saúde (81, I8\%) (Tabela 3).

Em relação à presença de depressão na amostra estudada, encontramos que $67,74 \%$ apresentaram algum nível de depressão, sendo que em 33,87\% dos pacientes a depressão era moderada ou grave, de acordo com o inventário de Beck. O escore médio do inventário de depressão de Beck encontrado foi 15,66.

Correlacionando-se a presença de depressão com as outras variáveis do estudo, encontramos que os fatos de não saber o diagnóstico $(p=0,008)$, não ter sido submetido a tratamento cirúrgico $(p=0,0$ | 3898$)$ e estar internado num hospital $(p=0,00 / 9)$ favoreceram níveis mais altos de depressão. Por outro lado, estar recebendo algum tratamento $(p=0,007)$ e acompanhamento ambulatorial $(p=0,0019)$ se correlacionaram negativamente com depressão. 


\section{Tabela 3 - Questionário estruturado para pacientes oncológicos em tratamento paliativo}

Atividades

Eu continuo trabalhando

Eu realizo minhas tarefas domésticas

Eu continuo aproveitando os prazeres da vida

Eumantenho meus hobbies/atividades de lazer

\section{Autonomia}

Consigo dirigir ou usar transporte público (ônibus, metrô, trem)

Sou auto-suficiente, consigo me vestir ou ir ao banheiro sozinho

Saúde

Eusinto dor

Eu sinto náuseas/enjôos ou vômitos (circule qual dos sintomas você sente)

Eu sinto falta de apetite

Eu sinto cansaço e/ou fraqueza

Eu sinto falta de ar

Eu tenho diarréía ou intestino preso (circule qual dos sintomas você sente)

Eutenho alterações no sono

\section{Escolha do Tratamento}

Eu gostaria de escolher meu esquema de tratamento

Eu sou capaz de escolher meu esquema de tratamento

A escolha do tratamento depende de: (coloque I para o item mais importante,

2 para o segundo item importante, e assim por diante, até o número 5)

Eficácia do tratamento

Qualidade de vida a curto e longo prazo

Complicações sobrea sua sexualidade Possíveis efeitos colaterais

Apoio - Eu me sinto satisfeito com o apoio de:

Parentes e/ou amigos

Aequipe médica

A equipe de enfermagem

Comunicação - Eu discuto com meu médico:

Minhas relações sociais

Meus problemas econômicos e profissionais

Meus problemasfamiliares

\section{Efeitos Psicológicos}

Eu me sinto calmo

Eu me sinto otimista

Eu me sinto triste

Sinto que estou no controle da situação

Eu tenho medo de morrer

\section{Não}

$\mathrm{N}(\%)$

53

$(85,48 \%)$

27

$(43,55 \%)$

34

$(54,84 \%)$

35

$(56,45 \%)$
As vezes

$\mathrm{N}(\%)$

5

$(8,07 \%)$

16
$(25,81 \%)$

$25,81 \%$
9

(14,52\%)

|1

(17,74\%)

8
$(12,91 \%)$
4
$(6,45 \%)$

(16,13\%)

20

$(32,26 \%)$

35

$(56,45 \%)$

32

$(51,61 \%)$

24

$(38,71 \%)$
43

43
$(69,35 \%)$

32

$(51,61 \%)$

20

$(33,90 \%)$

42
$(68,85 \%)$
45
$(73,77 \%)$

17

$(27,42 \%)$

11
$17,74 \%$

$(17,74 \%)$
8

$(12,91 \%)$

8

$(12,91 \%)$

6
$9,68 \%$

$(9,68 \%)$
10

(16,13\%)

12

(20,34\%)

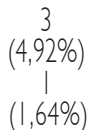

3
$(4,92 \%)$
$\mid$

$(1,64 \%)$

$40,32 \%$

$45,15 \%$

$1,61 \%$

$0 \%$
Sim

$\mathbf{N}(\%)$

4

(6,45\%)

19

(30,64\%)

19

(30,64\%)

16

$(25,81 \%)$

25

$(40,32 \%)$

48

$(77,42 \%)$

25

$(40,32 \%)$

16

$(25,81 \%)$

22

$(35,48 \%)$

30

$(48,39 \%)$

13

$(20,97 \%)$

20

$(32,26 \%)$

27

(45,76\%)

16

(26,23\%)

15

(24,59\%) 


\section{Discussão}

$\mathrm{Na}$ literatura brasileira ainda são poucos os estudos que abordam o tema sobre cuidados paliativos em pacientes com câncer. Assim, a necessidade de se caracterizar melhor este tipo de paciente, bem como o desconhecimento de suas necessidades, sua qualidade de vida e a presença de depressão motivaram a realização deste trabalho.

Em nossa amostra encontramos que a grande maioria de nossos pacientes (87, 1\%) tem conhecimento do diagnóstico de câncer, e este é um fator de importância por se correlacionar negativamente com a depressão. Gulinelli e colaboradores mostraram que $94,35 \%$ dos pacientes avaliados, por meio de entrevistas com 363 pessoas atendidas no ambulatório ou internadas na enfermaria de um serviço universitário de Clínica Geral, desejavam ser informados do diagnóstico de câncer ${ }^{15}$. Dado semelhante ao encontrado por Chacon et al. em um estudo com 1 I 8 adultos leigos, que mostrou o desejo de $80 \%$ a $90 \%$ de saberem a verdade no caso de estarem com câncer, mesmo quando a única alternativa se resumisse ao tratamento paliativo ${ }^{16}$. Suchman e Mattheus sugerem que a informação diminui o sentimento de isolamento do paciente e colabora para uma cooperação mútua na relação médico-paciente ${ }^{17}$.

A comunicação entre o médico e o paciente paliativo em nosso estudo foi pobre, pois aproximadamente $80 \%$ não conversavam com seus médicos acerca de outros temas além de sua condição de saúde e $50 \%$ dos pacientes referiram o desejo de maiores informações sobre sua condição. Diferindo do nosso achado, um estudo realizado sobre a comunicação médico-paciente paliativo encontrou que os pacientes abordavam de maneira equilibrada assuntos sobre sua doença e qualidade de vida (41\% e $48 \%$, respectivamente) $)^{18}$. Já os médicos abordam este tema em apenas $23 \%$ dos assuntos discutidos durante a consulta ${ }^{18}$. Detmar et al. mostraram que, na prática médica, a importância dada aos aspectos da qualidade de vida na decisão de (des)continuar o tratamento é menor que a sugerida na teoria ${ }^{19}$.

Encontramos que $61,22 \%$ dos pacientes não participaram da decisão de parar de tratar a doença com intuito curativo, o que pode refletir a falta de desejo $(68,85 \%)$ e capacidade $(73,77 \%)$ referida por eles de escolher seu esquema terapêutico. Ao contrário do encontrado por nós, Gulinelli et al. acharam que $58,5 \%$ das mulheres e 39,6\% dos homens desejavam opinar sobre o tratamento e um número ainda maior ( $86 \%$ das mulheres e $76,6 \%$ dos homens) gostaria de ser informado sobre as opções terapêuticas em caso de tumor ${ }^{15}$. Essa discordância dos resultados talvez se deva ao fato de que o estudo de Gulinelli et al. não foi conduzido com pacientes com câncer e sim com pessoas saudáveis às quais se expunha uma situação hipotética.

A depressão é uma doença cujas conseqüências emocionais e físicas apresentam grande impacto na qualidade de vida dos pacientes em tratamento paliativo. Mas, apesar da facilidade do tratamento e da boa resposta clínica ${ }^{7,20}$, esta doença ainda permanece subdiagnosticada nos pacientes oncológicos $8,9,10,11$. A depressão foi freqüente nos pacientes em cuidados paliativos que estudamos (67,74\%), e mais prevalente nos internados $(p=0,0019)$, nos que desconheciam seu diagnóstico $(p=0,008)$ e nos que não receberam nenhum tipo de tratamento oncológico $(p=0,007)$. Um estudo mostra que a depressão estará presente em $25 \%$ dos pacientes admitidos nas unidades de cuidados paliativos, enquanto na população geral este valor cai de $6 \%$ a $10 \%$. Breitbart et al. demonstraram que, em pacientes com câncer em estágio terminal internados, havia $16 \%$ de depressão grave, e em nosso estudo esse valor foi de $9,68 \%{ }^{21}$. Um dos fatores, conforme descrito por Lloyd et al. é o momento em que se avalia a depressão nestes pacientes que pode interferir nos resultados, pois em seu estudo a prevalência de depressão foi tanto maior quanto mais próximos do fim da vida estavam os pacientes ${ }^{22}$

Com relação ao fato de não ter recebido nenhum tratamento oncológico estar relacionado com depressão, Navari et al. mostraram que muitos pacientes com câncer avançado querem ser tratados mesmo que a chance de prolongar a vida seja pequena ${ }^{23}$. Essa preferência por uma terapia de prolongar a vida pode ser devida a um desejo do paciente de não desistir ou por um pobre entendimento de seu prognostico ${ }^{24}$.

Hall e colaboradores realizaram uma metanálise com 4 I estudos e concluíram que a satisfação do paciente está relacionada à quantidade de informação recebida e à participação no tratamento ${ }^{25}$. Não obstante estas falhas na condução do paciente paliativo, a maior parte dos estudados por nós $(93,33 \%)$ encontra-se satisfeita.

\section{ConClusão}

Este estudo revela que pacientes sob cuidados paliativos em nosso meio, apesar de geralmente satisfeitos com seu cuidado, relatam pobre comunicação com seu médico e apresentam uma alta taxa de depressão.

Saber o seu diagnóstico e ter recebido tratamento oncológico se correlacionam inversamente com a presença de depressão.

\section{Conflito de interesse: não há}

\section{SUMMARY}

\section{Awareness OF CANCER Diagnosis DOES NOT LEAD tO DEPRESSION IN PALLIATIVE CARE PATIENTS}

OBJECTIVE. Characterize the profile of patients under palliative care at this institution and evaluate the prevalence of depression in these patients.

METHODS. Sixty two cancer patients under palliative care, who had answered three questionnaires: one regarding their demographic characteristics, another to evaluate their quality of life and the Beck's depressioninventoryweresurveyed.

RESULTS. Of these patients, $68 \%$ presented with some degree of depression. Most of them were aware of their diagnosis (87. 1\%), did not talk to their physicians on other subjects but their disease (81.18\%), were satisfied with theirtreatment (93.33\%) and with the support they received (95.70\%). Pain, fatigue, weakness and sleep disturbances were the most frequently reported symptoms. There was a significant correlation between presence of depression and not knowing the diagnosis $(p=0.008)$, being admitted to the hospital $(p=0.0019)$ and not having ever received oncologic treatment.

CONCLUSION. Patients under palliative care at this institution, despite being satisfied with the treatment, reported poor communication with 
their physicians and presented with a high rate of depression. Awareness of their diagnosis and having received prior oncologic treatment $(p=0.07)$ correlated significantly and inversely with having depression. [Rev Assoc Med Bras 2006; 52(5): 298-303]

KEY wORDS: Cancer. Palliative care. Depression. Quality of life.

\section{REFERÊNCIAS}

I. Ministério da Saúde: Instituto Nacional de Câncer. Estimativas da Incidência e Mortalidade por Câncer no Brasil 2003. Disponível em: http:/ /www.inca.gov.br/estimativas/2003/. [citado 6 out 2004].

2. American Cancer Society. Cancer Statistics Presentation 2004. Disponível em: http://uww.cancer.org/downloads/PRO/Cancer\%20Statistics\%202004. ppt. [citado out 2004].

3. Gutierrez PL. O que é o paciente terminal? 200 I; [I]. Disponível em: http://www.admiror.com/sites/geyer/geyermed/n200 l09002.html. [citado 19 out 2004].

4. Melo AGC. Cuidados paliativos: histórico e filosofia. [I]. Disponível em: http://www.oncoguia.com.br/dor/0 I historico.asp. [citado 19 out 2004].

5. Lara-Munoz MC, Ponce de Leon S, Ramon de la Fuente J. Conceptualization and measurement of the quality of life of cancer patients. Rev Invest Clin 1995;47:3 I 5-27.

6. Lloyd-Williams M. Screening for depression in palliative care patients: a review. Eur J Cancer Care 200 I ; 0:3 I-5.

7. Lloyd-Williams M. Depression in advancer cancer: a hidden symptom. J R Coll Physicians Lond 200 I; 1: 175-6.

8. Rodin G, Voshart K. Depression in the medically ill: an overview. Am J Psychiatry 1 986; | 43:696-705.

9. Kathol RG, Noyes R Jr, Williams J, Mutgi A, Carroll B, Perry P. Diagnosing depression in patients with mental illness. Psychosomatics 1990;31:434-40.

10. Pirl W, Roth A. Diagnosis and treatment of depression in cancer patients. Oncology 1999; 1 3: 1 293-302.

II. Maguire P. Improving the detection of psychiatric problems in cancer patients. Soc Sci Med 1985;20:81 9-23.

12. Maguire P, Howell A. Improving the psychological care of cancer patients: Psychiatric aspects of physical disease. J R Coll Physicians Lond 1995; I:4 |-54

13. Mystakidou K, Tsilika E, Kouloulias V, Parpa E, Katsouda E, Kouvaris J, Vlahos L. The "Paliattive Care Quality of life Instrument (PQLI)" in terminal cancer patients. 2004; [I]. Disponível em: http://www.hqlo.com/ content/2/I/8. [citado 19 out 2004].
14. BeckAT, Beck SW. Screening depressed patients in family practice: a rapid technic. Postgrad Med 1972;52:8I-5.

15. Guinelli A, Aisawa RK, Konno SN, Morinaga CV, Costardi WL, Antonio $\mathrm{RO}$, et al. Desejo de informação e participação nas decisões terapêuticas em caso de doenças graves em pacientes atendidos em um hospital universitário. Rev Assoc Med Bras 2004;20:4I -6.

16. Chacon JP, Kobata CM, Lieberman SPC. A "mentira piedosa" para o canceroso. Rev Ass Med Bras 1995;41:274-6.

17. Suchman AL, Mattheus DA. What makes the patient-doctor relationship therapeutic? Exploring the connectional dimension of medical care. Ann Intern Med I 998; I 08: I 25-30.

18. Detmar SB, Muller MJ, Wever LD, Schornagel JH, Aaronson NK. Patientphysician communication during outpatient palliative treatment visits: an observational study. JAMA 200 I;285: I35 I-7.

19. Detmar SB, Aaronson NK, Wever LD, Muller M, Schornagel JH. How are you feeling? who wants to know? patients' and oncologists' preferences for discussing health-related quality-of-life issues. J Clin Oncol 2000; 1 8:3295301.

20. Barraclough J. $A B C$ of palliative care: Depression, anxiety and confusion. BMJ 1997;3 | 5(7| | 9): | 365-8.

21. Breitbart W, Rosenfeld B, Pessin H, Kaim M, Funesti-Esch J, Galietta M, et al. Depression, hopelessness, and desire and use of support services by cancer patients at Sydney hospitals. Aust N Z J Psychiatric 2000;284:2907-91।

22. Lloyd-Williams M. Depression in palliative care pacients: a prospective study. Eur J Cancer Care 200 I; I 0:270-4.

23. Navari RN, Stocking CB, Siegler M. Preferences of patients with advanced cancer for hospice care. JAMA;284:2449.

24. Voogt E. Attitudes of pacientes with incurable cancer toward medical treatement in the last phase of life. J Clin Oncol 2005;23:20 I 2-9.

25. Hall JA, Irish JT, Roter DL, Ehrlich CM, Miller LH. Gender in medical encounters: an analysis of physician and patient communication in primary care setting. Health Psychol 1994; 13:384-92.

Artigo recebido: 10/1 I/2005

Aceito para publicação: 03/06/2006 